\title{
Coronavirus Activates an Altruistic Stem Cell-Mediated Defense Mechanism that Reactivates Dormant Tuberculosis
}

\section{Implications in Coronavirus Disease 2019 Pandemic}

\author{
Lekhika Pathak, * Sukanya Gayan, * Bidisha Pal, *† Joyeeta Talukdar, * Seema Bhuyan, * Sorra Sandhya, * Herman Yeger, \\ Debabrat Baishya, ${ }^{\star \S}$ and Bikul Das*†
}

From the Department of Stem Cell and Infectious Diseases, * KaviKrishna Laboratory, Guwahati Biotech Park, Indian Institute of Technology, Guwahati, India; the Department of Stem Cell and Infection, ${ }^{\dagger}$ Thoreau Lab for Global Health, University of Massachusetts, Lowell, Massachusetts; the Department of Pediatric Laboratory Medicine, ${ }^{\ddagger}$ Hospital for Sick Children, Toronto, Ontario, Canada; and the Department of Bioengineering and Technology, ${ }^{\S}$ Gauhati University, Guwahati, India

\author{
Accepted for publication \\ March 25, 2021. \\ Address correspondence to \\ Bikul Das, M.D., Ph.D., \\ KaviKrishna Laboratory, \\ Guwahati Biotech Park, IIT- \\ Guwahati, Assam 781039, \\ India. E-mail: bdas@ \\ kavikrishnalab.org.
}

\begin{abstract}
We postulate that similar to bacteria, adult stem cells may also exhibit an altruistic defense mechanism to protect their niche against external threat. Herein, we report mesenchymal stem cell (MSC)-based altruistic defense against a mouse model of coronavirus, murine hepatitis virus-1 (MHV-1) infection of lung. MHV-1 infection led to reprogramming of $\mathrm{CD} 271^{+} \mathrm{MSC}$ in the lung to an enhanced stemness phenotype that exhibits altruistic behavior, as per previous work in human embryonic stem cells. The reprogrammed MSCs exhibited transient expansion for 2 weeks, followed by apoptosis and expression of stemness genes. The conditioned media of the reprogrammed MSCs exhibited direct antiviral activity in an in vitro model of MHV-1-induced toxicity to type II alveolar epithelial cells by increasing their survival/proliferation and decreasing viral load. Thus, the reprogrammed MSCs can be identified as altruistic stem cells (ASCs), which exert a unique altruistic defense against MHV-1. In a mouse model of MSC-mediated Mycobacterium tuberculosis (MTB) dormancy, MHV-1 infection in the lung exhibited 20fold lower viral loads than the MTB-free control mice on the third week of viral infection, and exhibited six-fold increase of ASCs, thereby enhancing the altruistic defense. Notably, these ASCs exhibited intracellular replication of MTB, and their extracellular release. Animals showed tuberculosis reactivation, suggesting that dormant MTB may exploit ASCs for disease reactivation. (Am J Pathol 2021, 191: 1255-1268; https://doi.org/10.1016/j.ajpath.2021.03.011)
\end{abstract}

The severe acute respiratory syndrome coronavirus 2 (SARSCoV-2)-mediated coronavirus disease 2019 (COVID-19) pandemic demonstrates the ability of an emerging virus to generate chaos in our modern health care system and a severe strain on global economics. Postpandemic, the SARS-CoV-2 might activate dormant bacterial infections in the long term. As per prior history, tuberculosis is one of the key bacterial infections affected by viral pandemics. ${ }^{1-5}$ Strikingly, onequarter of the world population is already infected with dormant tuberculosis (TB) (https://www.who.int/news-room/ fact-sheets/detail/tuberculosis, last accessed October 14,
2020). If SARS-CoV-2 infects these dormant TB populations, it may cause severe impact on global health and economics by causing both COVID-19 and dormant TB reactivation. Thus, there is an urgent need to study the

\footnotetext{
Supported by the Laurel Foundation grants (B.D.), the KaviKrishna Foundation (Assam, India) grants KKL/2018-4_MHV-1 (L.P., S.G., S.B., J.T., and S.S.), the Department of Biotechnology India grant BT/PR22952/ NER/95/572/2017 (B.D.), and the KaviKrishna USA Foundation grants KKL/2018-4_MHV-1 (B.D. and B.P.).

Disclosures: None declared.
} 
association of COVID-19 with dormant TB reactivation to avoid a later global TB pandemic.

Viral infection, such as the influenza virus or SARS-CoV-1, is known to cause transient immune suppression that leads to reactivation of dormant bacterial infection. ${ }^{4,6,7}$ In 1918, the Spanish flu pandemic led to the rise of pulmonary TB incidence. ${ }^{2,8}$ Influenza in patients with TB led to the highest death rate. ${ }^{8,9}$ In the year 2009 during the influenza A (H1N1) pandemic, a worse prognosis of influenza was observed in patients with TB or multidrug-resistant TB. ${ }^{10,11}$ Interestingly, SARS-CoV-1 and Middle East respiratory syndrome coronavirus-infected patients were reported to develop pulmonary TB. ${ }^{4,5}$ In a mouse model, influenza A virus causes rapid development of pulmonary TB lesions ${ }^{12}$ and an increase in the mycobacterium load in the liver. ${ }^{13}$ Another mouse model of influenza A virus and Mycobacterium tuberculosis (MTB) co-infection leads to enhanced MTB growth by a type I interferon signaling pathway. ${ }^{6}$ However, severe inflammation in the lung is a common outcome of coronavirus infection, ${ }^{14}$ a symptom that is also commonly observed in TB patients. ${ }^{15}$ Thus, it is possible that coronavirus infection-causing inflammation may also reactivate dormant MTB (dMTB) in the lung, which has not yet been studied.

Several studies have investigated TB dormancy in the adult stem cell niches. ${ }^{16-18}$ These stem cells reside in the bone marrow (BM) niche ${ }^{19}$ and in the area of inflammation. ${ }^{20}$ A rare fraction of cells in the BM, the $\mathrm{CD} 271^{+}$ BM-mesenchymal stem cells (CD271 ${ }^{+}$BM-MSCs) has been identified as the potential niche for $\mathrm{dMTB}$ in mice and in successfully treated TB patients. ${ }^{17}$ In this stem cell niche, MTB remains dormant, maintaining reactivation potential. More importantly, we have developed a mouse model of stem cell-mediated MTB dormancy. ${ }^{17}$ Briefly, streptomycindependent mutant $18 \mathrm{~b}$ strain-infected mice exhibit lung infection following 3 weeks of streptomycin treatment. These mice develop granulomas, and acquire humoral immunity against the bacteria. Following streptomycin starvation for 6 months, the bacteria acquire a nonreplicating status. These bacteria can primarily be detected in the CD271 ${ }^{+}$MSCs of $\mathrm{BM}$, but a few are also present in the CD271 ${ }^{+}$MSCs of lung. ${ }^{17}$ Furthermore, MTB harboring CD271 ${ }^{+}$MSCs reside in the hypoxic niche of BM. ${ }^{18}$ Notably, in this model, the significant increase of MTB-colony-forming units (CFUs) in the non-CD271 ${ }^{+}$MSC compartment of lung can be used as a sign of MTB reactivation. Additionally, the potential reprogramming of the $\mathrm{CD} 271^{+}$MSCs to enhanced stemness phenotype having altruistic behavior ${ }^{21,22}$ can also be studied in this model. Briefly, the enhanced stemness is a transient phenotype of stem cells characterized by their ability to not only maintains stemness, but also secrete cytoprotective agents during extreme oxidative stress or inflammation. ${ }^{21,22}$ The enhanced stemness phenotype was characterized by exposing human embryonic stem (ES) cells to the microenvironment of oxidative stress. In such hostile microenvironment, some of the ES cells underwent enhanced stemness reprogramming by activating a hypoxia-inducible factor
(HIF)-2 $\alpha$ stemness pathway that altered the p53/mouse double minute 2 homolog oscillation to a transient state of low p53. The low p53 state permits these cells to maintain a state of enhanced stemness (state of self-renewal and selfsufficiency) in the microenvironment oxidative stress. ${ }^{22}$ While in such hostile microenvironment, naive stem cells usually undergo differentiation/apoptosis, ${ }^{21}$ and the reprogrammed ES cells not only maintain stemness, but also selfrenew. Thus, these reprogrammed ES cells gain fitness. However, instead of becoming the dominant subpopulation, these cells sacrifice their self-fitness to enhance the fitness of neighboring cells under stress by secreting glutathione, an intracellular antioxidant that cells do not actively secrete. Moreover, the cultured supernatant of these reprogramed ES cells defend hematopoietic and mesenchymal stem cells from oxidative stress-induced differentiation/apoptosis. This is considered an altruistic behavior, a form of stem cell altruism, ${ }^{23}$ as these glutathione-secreting ES cells sacrifice their newly acquired fitness by returning to basal state of p53/ MDM2 oscillation, leading to p53-mediated apoptosis/differentiation. $^{22}$ Hence, these transient stem cells acquiring enhanced stemness phenotype can be termed as altruistic stem cells (ASCs) as opposed to competitive stem cells, which eliminate weak neighbors during stress. ${ }^{21,24,25}$ The underlying significance of further studying stem cell altruism is that it is equivalent to an innate defense mechanism of cytoprotection, because similar to microbial altruistic defense against stress, ${ }^{26,27}$ ASC mechanisms may also have evolved as a potent stem cell defense of cytoprotection from stressful microenvironment-induced differentiation/apoptosis. ${ }^{21}$ Developing an experimental model to study ASC-based defense will strengthen our growing understanding of stem cell niche defense against pathogen invasion, and provide novel insight about how pathogens, such as dMTB, may hijack this defense for their own benefit. Herein, we hypothesize that following viral infection, lung MSCs present in the alveolar stem cell niche ${ }^{28,29}$ may also exhibit altruistic defense by reprogramming to ASCs. The dormant MTB hiding in the intracellular compartment of these MSCs may then exploit the ASC reprograming for TB reactivation. ${ }^{25}$

Therefore, this mouse model of stem cell-mediated MTB dormancy was used to find out if coronavirus can reactivate dMTB by inducing MSC to ASC reprogramming. A mouse coronavirus strain, the murine hepatitis virus-1 (MHV-1), that represents clinically relevant human coronavirus SARSCoV-1 infection, was used. ${ }^{30-32}$ MHV-1 infection causes acute lung inflammation by inducing acute respiratory infection within 2 to 4 days in C57BL/6 mice by increasing viral load. Animals exhibit an elevated level of proinflammatory cytokines, such as tumor necrosis factor (TNF)- $\alpha$, during 2 to 14 days postinfection ${ }^{30,33}$ and then fully recover. ${ }^{30}$ Therefore, the MHV-1-infected C57BL/6 mice can be utilized for MTB reactivation study.

Herein, MHV-1 infection was shown to cause dMTB reactivation in the mouse model of stem cell-mediated MTB dormancy. Furthermore, MHV-1 was found to 
reprogram the $\mathrm{CD} 271^{+}$MSCs to an enhanced stemness or ASC phenotype, and culture supernatant of these reprogrammed MSCs was found to enhance the survival/proliferation of MHV-1-infected type II alveolar epithelial (ATII) cells. After 2 weeks of expansion in the MHV-1-infected lung, these reprogrammed MSCs activated p53 upstream genes involved in apoptosis/differentiation, and underwent apoptosis, thus sacrificing self-fitness while enhancing the fitness of the alveolar epithelial cells, a behavior akin to ES cells exhibiting enhanced stemness or ASC phenotype. Thus, these findings may facilitate the study of proposed ASC-mediated altruistic defense of stem cell niche. ${ }^{21,25,34}$ The remarkable significance of this study is that although MHV-1 infection causes dMTB reactivation, it also activates an ASC-based innate defense mechanism against the virus and that could be further explored to develop therapeutics to target coronavirus.

\section{Materials and Methods}

\section{Mycobacterium Strain and Growth Condition}

All of the necessary experimental procedures were approved and undertaken inside Biosafety Cabinet class II facility in accordance with guidelines of Institutional Bio-safety Committee of KaviKrishna Laboratory. Streptomycinauxotrophic mutant MTB strain18b (gifted by Prof. Stewart T. Cole, Ecole Polytechinque Federale de Lausanne, Lausanne, Switzerland) was cultured in BBL Middlebrook 7H9 broth with glycerol (BD Biosciences, Gurugram, Haryana, India; number 221832) along with $50 \mu \mathrm{g} / \mathrm{mL}$ of streptomycin sulfate. It was maintained at $37^{\circ} \mathrm{C}$ and $5 \%$ $\mathrm{CO}_{2}$ with occasional shaking until the midlogarithmic phase was reached, OD approximating to 1 .

\section{Development of Stem Cell-Mediated Mouse Model of MTB Reactivation}

All of the necessary experimental procedures were undertaken in accordance with approvals of Institutional Animal Ethics Committee, Gauhati University, and Institutional Ethics Committee, KaviKrishna Laboratory. The 6- to 8week-old C57BL/6 female mice were obtained from National Institution of Nutrition (Hyderabad, India) and were maintained in the animal house of Gauhati University at pathogen-free condition, as previously described. ${ }^{17}$ The mouse model of MTB dormancy was developed in 6 months. Briefly, streptomycin-auxotrophic mutant MTB strain 18b cell suspension was prepared in phosphate-buffered saline-Tween $80(0.05 \%)$, sonicated for 15 seconds, and intravenously injected with $2 \times 10^{6} \mathrm{CFUs}$ per mouse. Initially for 3 weeks, streptomycin was administered $(3 \mathrm{mg} /$ mouse in $200 \mu \mathrm{L}$ of normal saline) daily for establishing infection. Then, no streptomycin treatment was provided for 6 months to establish bacterial dormancy. Following 6 months of streptomycin starvation, lung tissue was dissociated;
$\mathrm{CD} 271^{+} / \mathrm{CD} 45^{-}$MSCs and non-CD271 ${ }^{+}$cells were isolated by magnetic sorting, as previously explained. ${ }^{18}$ The magnetically sorted CD271 ${ }^{+}$MSCs and non-CD271 ${ }^{+}$cells were subjected to MTB-CFU assay. Consistent with previous findings, ${ }^{17}$ a small number of MTB-CFUs $(38 \pm 10$ MTBCFU/lung) were obtained only in $\mathrm{CD} 271^{+}$MSCs. Thus, mouse model of MTB dormancy was developed. Streptomycin was injected intraperitoneally ( $3 \mathrm{mg} /$ mouse in $200 \mu \mathrm{L}$ of normal saline) daily for 3 weeks ${ }^{17}$ with or without MHV-1 infection $^{30,32}$ to develop a mouse model of reactivation. In a separate experiment, streptomycin-treated mice were also treated with immunosuppressive agents dexamethasone $(0.08$ $\mathrm{mg} /$ day, 6 times a week) or amino guanidine $(2.5 \% \mathrm{wt} / \mathrm{vol}$ in drinking water) to cause dMTB reactivation. ${ }^{35}$ The treated mice were observed for stipulated period of time, and then sacrificed to collect lung tissues. The MTB-CFU of lung was evaluated for MTB reactivation.

\section{Isolation and Culture of $\mathrm{CD} 271^{+} \mathrm{MSCS}$}

The mouse lung tissues were dissociated using collagenase/ lipase and then cells were subjected to magnetic sorting to isolate $\mathrm{CD}_{271^{+}}$MSCs, as explained previously. ${ }^{17,18}$ Briefly, CD $45^{-}$cells were magnetically sorted using Ter119/CD45 ${ }^{-}$depletion kit (number 19771; Stem Cell Technologies, Vancouver, BC, Canada). Next, the CD45 cells were subjected to magnetic sorting for isolation of CD271 ${ }^{+}$cells. First, mouse CD271 antibody (mouse clone ME20.4; catalog number Ab8877; Abcam, Cambridge, MA) was phycoerythrin conjugated by SiteClick antibody labeling kit (catalog number S10467; Life Technologies, Grand Island, NY). Next, the EasySep phycoerythrin sorting kit (catalog number 18554; Stem Cell Technologies) was used to isolate $\mathrm{CD} 271^{+}$cells from the $\mathrm{CD} 45^{-}$cell population. For MTB-CFU assay, cells were cultured for 4 to 8 hours in serum-free media without any growth factor. For cytoprotective assay, cells were isolated on day 8 following infection and cultured $\left(1 \times 10^{7}\right.$ cells $\left./ \mathrm{mL}\right)$ in Dulbecco's modified Eagle's medium/F12 media without serum for 24 hours to obtain the conditioned media (CM).

\section{MTB-CFU Assay}

The MTB-CFU assay was performed as previously described. ${ }^{17,18}$ For the whole lung MTB-CFU, the lung tissue was aseptically removed from sacrificed mice and homogenized in phosphate-buffered saline with $0.05 \%$ Tween 80, then subjected to CFU assay. ${ }^{18}$ For the MTBCFU assay of immunomagnetically sorted cells, cells were isolated by dissociating lung tissue using collagenase/ lipase. ${ }^{17}$ The pellet was lysed with $1 \mathrm{~mL}$ of $0.1 \%$ Triton X100 for 15 minutes and vortexed for 30 seconds, and serial 10-fold dilution was prepared in Middlebrook 7H9 broth. The diluted whole lung or cell lysate was then separately plated onto Middlebrook 7H10 agar plates (BD Biosciences; number 295964) along with streptomycin $(50 \mu \mathrm{g} / \mathrm{mL})$ for 
the growth of $\mathrm{m} 18 \mathrm{~b}$ strain. The agar plates were incubated for 3 to 4 weeks at $37^{\circ} \mathrm{C}$ and $5 \% \mathrm{CO}_{2}$, and $\mathrm{CFUs}$ were counted. CFUs were plotted as the means of $\log 10 \mathrm{CFUs}$ per lung or per $10^{7}$ cells.

\section{Real-Time Quantitative PCR Assay}

To isolate the mRNA from bacterial infected CD271 MSCs, we used the $\mu$ MACS technology (Miltenyi Biotec, Bergisch-Gladbach, Germany), as described previously. ${ }^{17}$ Briefly, the mRNA is isolated in a magnetic column using super paramagnetic Oligo (dT) Microbeads, which target the poly RNA tail of mammalian RNA. The mRNA gets attached to the magnetic column, whereas the bacterial and stem cell DNA remained in the passed-through lysate. Then, the mRNA was converted to cDNA in a heated magnetic bar, as per manufacturer's instruction, and cDNA was subjected to real-time quantitative PCR analysis using the TaqMan Gene expression assay (Miltenyi Biotech). RNA quantification was done using the $\Delta \Delta \mathrm{Ct}$ method by using the SDS software version 2.2.1. ${ }^{17}$ The following TaqMan primers were used: mouse: CD271 (Mm 00446296_m1), CD45 (Mm 01293575_m1), CD44 (Mm01277163_m1), HIF-2 $\alpha$ (Mm01236112_m1), ATP binding cassette subfamily $G$ member 2 (ABCG2) (Mm00496364_m1), octamer-binding transcription factor 4 (Mm00658129_g1), Nanog (Mm02019550_s1), sex-determining region Yrelated HMG box 2 (Mm03053810_s1), HIF-1 $\alpha$ (Mm00468869_m1), p53 (Mm01731290_g1), p21 (Mm01332263_m1), MDM2 (Mm01233136_m1), glutamate cysteine ligase (GCL; Mm00802655_m1), CD73 (Mm00501915_m1), and glyceraldehyde-3-phosphate dehydrogenase (Mm99999915_g1).

\section{MHV-1 Infection into Mice}

Parental virus strain MHV-1 was obtained from ATCC (Manassas, VA) and cultured inside the BSC class II facility at KaviKrishna Laboratory in accordance with approval of Institutional Bio-safety Committee and Institutional Ethics Committee of KaviKrishna Laboratory. The animal protocol was approved by the Institutional Ethics Committee of KaviKrishna Laboratory and Gauhati University. MHV-1 was propagated on L2 (ATCC HCCL-149) cells, purified by sucrose gradient centrifugation, and titrated by end point dilution assay on L2 cells to determine titer of the viral stock, as previously described. ${ }^{36}$ Mice [C57BL/6 mice previously infected with the mutant $18 \mathrm{~b}$ MTB strain, followed by 6 months of streptomycin starvation, or control group (25- to 26-week-old healthy mice)] were intranasally infected with 5000 plaque-forming units (PFUs) of MHV-1 per mouse. ${ }^{30,32}$ Briefly, animals were anesthetized with ketamine intraperitoneally and then $50 \mu \mathrm{L}$ of MHV-1 in phosphate-buffered saline was administered intranasally; this dose is known to cause acute respiratory infection in this strain of mice. ${ }^{31,33}$ Evaluation of lung infection was done by viral load study and measurement of TNF- $\alpha$ on days 0 and 6 , as described. ${ }^{30,33}$

\section{Viral PFU Assay}

The viral PFU assay was performed as previously described. ${ }^{36}$ Animals were euthanized at specific times, and the whole lungs were harvested. The lung tissue was homogenized as described, ${ }^{17}$ and the supernatant was stored at $-80^{\circ} \mathrm{C}$ before further analysis. One day before the formation of the PFU assay, L2 cells were seeded so that the cell density could reach confluence on the day of PFU assay. The serial dilution of lung homogenate derived supernatant was loaded on the L2 monolayer cells and incubated for 1 hour at $37^{\circ} \mathrm{C}$. The inoculum was removed, and it was overlayered with methylcellulose media and incubated for 48 hours at $37^{\circ} \mathrm{C}$. The plaques were then counted, and the virus titer was calculated using the following formula: titer $(\mathrm{PFU} / \mathrm{mL})=[($ number of plaques $/$ well $) /($ volume of inoculum/well)] $\times$ dilution factor.

\section{Enzyme-Linked Immunosorbent Assay for TNF- $\alpha$ Evaluation}

This was done by using an Elisa Kit (number MTA00B) of R\&D Systems (Minneapolis, MN), as described previously. ${ }^{22}$ Briefly, whole lungs were harvested and homogenized in Dulbecco's modified Eagle's medium/F12 medium supplemented with protease inhibitor mix (Sigma Aldrich, St. Louis, MO). Lung homogenates were centrifuged, and $50 \mu \mathrm{L}$ of supernatant was added to the wells of the enzymelinked immunosorbent assay plate, covered, and incubated at room temperature for 2 hours. Each well was aspirated and washed for five times, as mentioned in the kit. A total of $100 \mu \mathrm{L}$ of mouse TNF- $\alpha$ conjugate to horseradish peroxidase was added to each well and covered with an adhesive strip. It was then incubated for 2 hours at room temperature and then aspirated and washed. The plates were developed for 30 minutes using $100 \mu \mathrm{L}$ of tetramethylbenzidine plus hydrogen peroxide. Plates were read at $450 \mathrm{~nm}$ using iMark Microplate Absorbance Reader (Biorad, Gurgaon, India).

\section{Cellular Caspase-3/7 Assay}

The caspase- $3 / 7$ activity assay was performed as previously described. ${ }^{22,37}$ Briefly, the day 8 and day $20 \mathrm{CD} 271^{+}$MSCs were isolated from the MHV-1 alone (with streptomycin), dMTBMHV-1, dMTB alone (with streptomycin), and streptomycin alone (control mice treated with streptomycin) group ( $n=5$ for each group). Then, isolated CD271 ${ }^{+}$ MSCs were cultured $\left(2 \times 10^{4}\right.$ cells $\left./ \mathrm{mL}\right)$ in serum-free Dulbecco's modified Eagle's medium for 8 hours and subjected to a colorimetric assay of caspase-3/7 activity. ${ }^{22,37}$ 


\section{In Vitro Cytoprotection Assay Against MHV-1 Infection}

The ATII cells of healthy C57BL/6 mice were first isolated as previously described ${ }^{38}$ by isolating $\mathrm{CD} 45^{-}$and epithelial cell adhesion molecule ${ }^{+}$cells using immunomagnetic sorting. ${ }^{39}$ The isolated cells were cultured in the $70 \%$ rat tail collagen (Sigma, St. Louis, MO) and 30\% Matrigel (BD Biosciences) coated 6 -well plates $\left(2 \times 10^{5}\right.$ cells/well $)$ using Dulbecco's modified Eagle's medium/F12/10\% fetal bovine serum and $10 \mathrm{ng} / \mathrm{mL}$ mouse keratinocyte growth factor with penicillin/streptomycin antibiotics. The cells were infected with MHV-1 with a multiplicity of infection of $1: 5$, as described, ${ }^{38}$ and then treated for 48 hours with the CM obtained from the lung CD271 ${ }^{+}$MSCs collected from the three groups (dMTB alone, MHV-1 alone, and the dMTBMHV-1 group). The $\mathrm{CM}$ was collected from in vivo $\mathrm{CD} 271^{+}$MSCs by a previously described method, ${ }^{22}$ where the isolated CD271 $1^{+}$MSCs $\left(1 \times 10^{7}\right.$ cells $\left./ \mathrm{mL}\right)$ were cultured in vitro for 24 hours, and then CM was filtered through a $0.2-\mu \mathrm{m}$ filter. The filtered CM was added to ATII cells. After 48 hours of growth, cytoprotection was measured by the reduction in viral load (viral plaque assay), cell survival (trypan blue assay), and cell proliferation (alamar blue assay), as previously described. ${ }^{40,41}$ For the cell proliferation assay, the viral infected cells were continuously grown for 6 days, and then alamar blue assay was performed every alternate day to measure proliferation, as described. $^{37}$

\section{Statistical Analysis}

Statistical analysis was performed using GraphPad Prism version 4.0 (Hearne Scientific Software, Chicago, IL). $t$-test was used for comparison with Newman-Keul post hoc test. Gene expression was analyzed using one-way analysis of variance with the Dunnett post hoc test. Data are expressed as means \pm SEM $(* P<0.05, * * P<0.01, * * * P<0.001$, and $* * * * P<0.0001)$.

\section{Results}

\section{MHV-1 Infection Leads to Reactivation of dMTB in C57BL/6 Mice}

To test whether MHV-1 infection may reactivate dMTB intracellular to MSCs in the lung MHV-1 $\left(5 \times 10^{3} \mathrm{PFU}\right)$ was intranasally administered to $\mathrm{C} 57 \mathrm{BL} / 6$ mice $(n=25),{ }^{30,32}$ with lung CD271 $1^{+}$MSCs, that contained streptomycin-dependent mutant MTB strain of $18 \mathrm{~b}$ in nonreplicating dormant state for 6 months (Figure 1A). ${ }^{17,42}$ Simultaneously, mice were also injected with streptomycin as the mutant $18 \mathrm{~b}$ strain requires the antibiotic to replicate. ${ }^{42}$ The study was ended after 3 weeks, because 3 weeks of streptomycin is sufficient to establish lung infection ${ }^{17} ; 2$ weeks after MHV-1 infection, the C57BL/6 mice recover from viral pneumonitis, ${ }^{30,32}$ which makes extending the

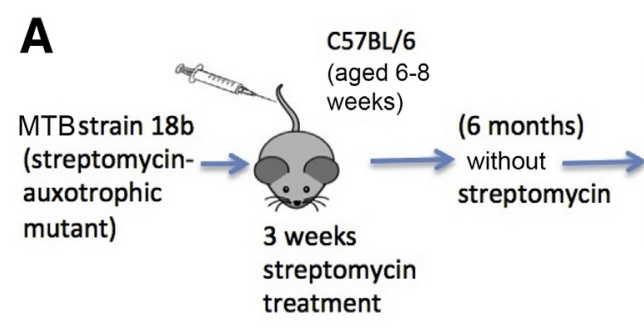

B

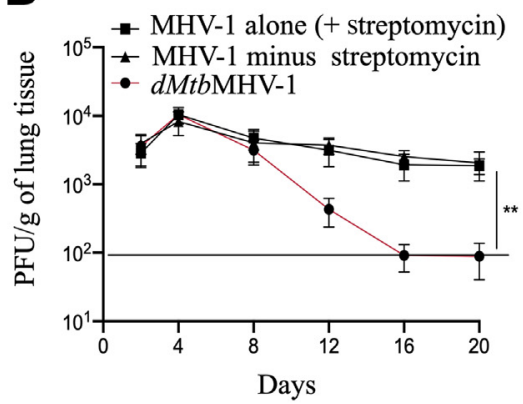

\section{C}

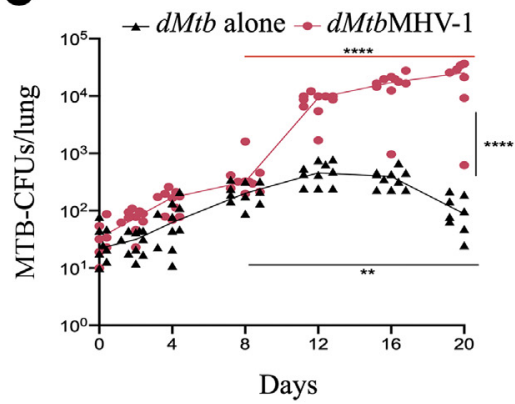

MHV-1

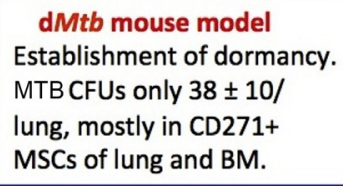

MSCs of lung and BM.

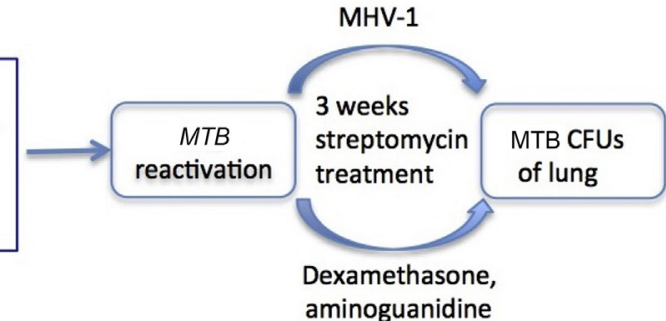

D

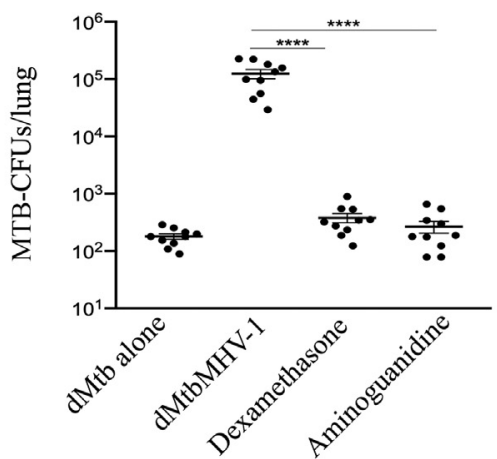

Figure 1 Murine hepatitis virus-1 (MHV-1) infection reactivates dormant Mycobacterium tuberculosis (MTB) in the lungs. A: Schematic of producing the dormant MTB (dMTB) mouse model, and the experimental design for dMTB reactivation. B and C: Viral load [plaque-forming unit (PFU)] and MTB-colonyforming units (MTB-CFUs) in the lungs from MHV-1-infected C57BL/6 mice at various times after infection. Solid line in B represents limit of detection. D: MTB-CFUs in the lungs of mice from day 30 after MHV-1 infection, or day 30 after treatment with dexamethasone or aminoguanidine. All of the mice were treated with daily streptomycin 6 days per week for 3 weeks. Data represent means \pm SEM (B-D). $n=4$ independent experiments (B-D). ${ }^{* *} P<0.01$, $* * * * P<0.0001$ ( $t$-test). dMTB alone, mice harboring dMTB intracellular to lung and bone marrow (BM) CD271 ${ }^{+}$MSCs, treated with streptomycin; dMTBMHV1, dMTB harboring mice infected with MHV-1 and treated with streptomycin; MHV-1 alone, mice infected with MHV-1 and treated with streptomycin; MHV-1 minus streptomycin, mice infected with MHV-1, not treated with streptomycin. 
study to third week ethical. Thus, the study was ended on 20th day following MHV-1 infection. During the 20 days of MHV-1 infection, animals $(n=5)$ were sacrificed at stipulated time, lungs were obtained, and the viral titers as well as MTB-CFUs in the lung tissues were evaluated (Figure 1, $\mathrm{B}$ and $\mathrm{C}$ ). TNF- $\alpha$ was measured in the animals' blood on day 2 of MHV-1 infection to confirm MHV-1-induced immune response. ${ }^{30}$ First, the viral infection was evaluated in mice, as shown in Figure 1B. MHV-1-infected mice were used as the control. In a subset of mice from the control group, streptomycin was also added to determine whether addition of streptomycin could affect viral load. MHV-1 infection in both the control groups [MHV-1 minus streptomycin (not treated with streptomycin) and MHV-1 alone (treated with streptomycin)] led to an increase in viral titers in the mouse lung for the first 4 days, and then gradually decreased within next 2 weeks (Figure 1B), which is consistent with the previous results from C57BL/6 mice. ${ }^{32}$ Notably, streptomycin had no effect on viral titers. MHV-1-infected mice with dMTB and treated with streptomycin (henceforth, dMTBMHV-1 group) showed an increase in viral load for the first 4 days similar to control, but then rapidly subsided at the end of 2 weeks, where the viral load was 20-fold less than control group $(P<0.004)$ (Figure 1B). The TNF- $\alpha$ levels in both the groups were increased by threefold to fourfold $(P<0.03 ; n=4)$ on day 2 (dMTB, $32.5 \pm 11.4$ versus $7.3 \pm 4.5 \mathrm{pg} / \mathrm{mL}$; dMTBMHV-1, $36.5 \pm 14.7$ versus $9.5 \pm 3.6 \mathrm{pg} / \mathrm{mL}$ ). These data suggest that MHV-1 infection activated viral replication, and associated immune response in both groups, although viral load decreased in MTB-infected versus noninfected mice.

Second, MTB-CFUs were evaluated in the lungs of dMTB alone versus dMTBMHV-1 group. Both the groups $(n=10$ mice in each group) received daily streptomycin treatment for mutant strain to replicate. By 8 days of infection, MTB-CFUs were sixfold to sevenfold greater than the MTB-CFU before the start of the infection in both the groups $(P<0.01)$ (Figure $1 \mathrm{C})$. Between days 8 and 20 of postinfection, MTB-CFUs increased by 110 -fold $(P<0.0001)$ in the dMTBMHV-1 group but decreased by 2.1 -fold $(P<0.004)$ in the dMTB alone group. These results indicate that MHV-1 infection not only initiated but also sustained the MTB reactivation process following streptomycin treatment. In contrast, MTB reactivation did not occur in the dMTB alone group as the streptomycin treatment alone failed to increase MTB-CFUs, even after 3 weeks of treatment. More importantly, 630-fold increase was observed in MTB-CFU between days 0 and 20 in the dMTBMHV-1 group, which is equivalent to a 1000-fold increase in MTB-CFU in a dexamethasone- and aminoguanidine-induced MTB reactivation model. ${ }^{35}$

Therefore, next the MHV-1-induced reactivation of the dMTB was compared with dexamethasone or aminoguanidine. These have been found to reactivate dMTB in a drug-induced Cornell model by suppressing the immune system. ${ }^{35}$ To compare the reactivation of MHV-1 versus these immunosuppressive agents, dMTB harboring mice were treated with either of these two drugs and streptomycin daily for a month, and the lung MTB-CFUs were quantified. Next, the MTB-CFU levels of these two drug treated mice were compared with the MTB-CFU obtained from 1 month after MHV-1-infected mice. MTB-CFUs increased by threefold to fourfold $(P=0.06 ; n=4)$ following either dexamethasone or aminoguanidine treatment, which was 400 -fold lower than the MHV-1 infection group $(P<0.0001$, $n=4$ ) (Figure 1D). Thus, MHV-1 infection was superior to dexamethasone or aminoguanidine to induce reactivation in our model of dMTB. These results also indicate that mechanisms other than immunosuppression may underlie MHV-1 infection-mediated disease reactivation.

\section{MHV-1 Infection Causes Expansion of Lung CD271 ${ }^{+}$ MSCs and Extracellular Pathogen Release}

In this mouse model of MTB dormancy, dMTB remains dormant in $\mathrm{CD} 271^{+}$MSCs of bone marrow and lung. We hypothesized that during dMTB reactivation, the bacteria transfer from MSCs to non-MSC compartments in the lung. ${ }^{17}$ Therefore, the lung CD271 ${ }^{+}$MSCs were analyzed for intracellular MTB replication and release into the extracellular space. Lung CD271 ${ }^{+}$MSCs were obtained by immunomagnetic sorting of $\mathrm{CD} 271^{+} / \mathrm{CD} 45^{-}$mononuclear cells after a stipulated period of MHV-1 infection. Flow cytometry was used to confirm $90 \%$ purity of these immunomagnetic sorted $\mathrm{CD} 271^{+}$MSCs, as previously described. ${ }^{17,18}$ Viable cell count was obtained by trypan blue to evaluate expansion of these cells in vivo in the stipulated period. The dMTB group served as control. Possible CD271 ${ }^{+}$MSC expansion was studied in mice infected with MHV-1 to evaluate a potential effect of this virus on MSCs of lung. Next, the sorted cells were cultured in serum-free media for 8 hours to evaluate both the intracellular and the extracellular release of MTB by measuring MTB-CFUs in the cell pellet and supernatant, respectively. The dMTBMHV-1 group showed a transient 12-fold $(P<0.001)$ (Figure $2 \mathrm{~A}$ ) expansion of $\mathrm{CD} 271^{+}$MSCs between days 0 and 8. Strikingly, the MHV-1 infection alone group exhibited a 4.5 -fold $(P<0.01)$ expansion of CD271 ${ }^{+}$ MSCs between the same period; however, the expansion was sixfold lower than the dMTBMHV-1 group (Figure 2A). However, the dMTB alone group showed no expansion of the MSCs (Figure 2A). Notably, in the dMTBMHV-1 group, along with MSC expansion, there was a corresponding 27 -fold $(P<0.001)$ (Figure $2 \mathrm{~B})$ increase in the number of intracellular MTB-CFUs. During this period (days 0 to 8), the supernatant of dMTBMHV-1 group did not exhibit marked increase in extracellular MTB-CFUs compared with dMTB control (Figure 2C). However, in the next 4 days (ie, between days 8 and 12), the supernatant showed a 40-fold increase of MTB-CFU in dMTBMHV-1 group $(P<0.001)$ (Figure $2 \mathrm{C})$. Thus, MTB transfer from 

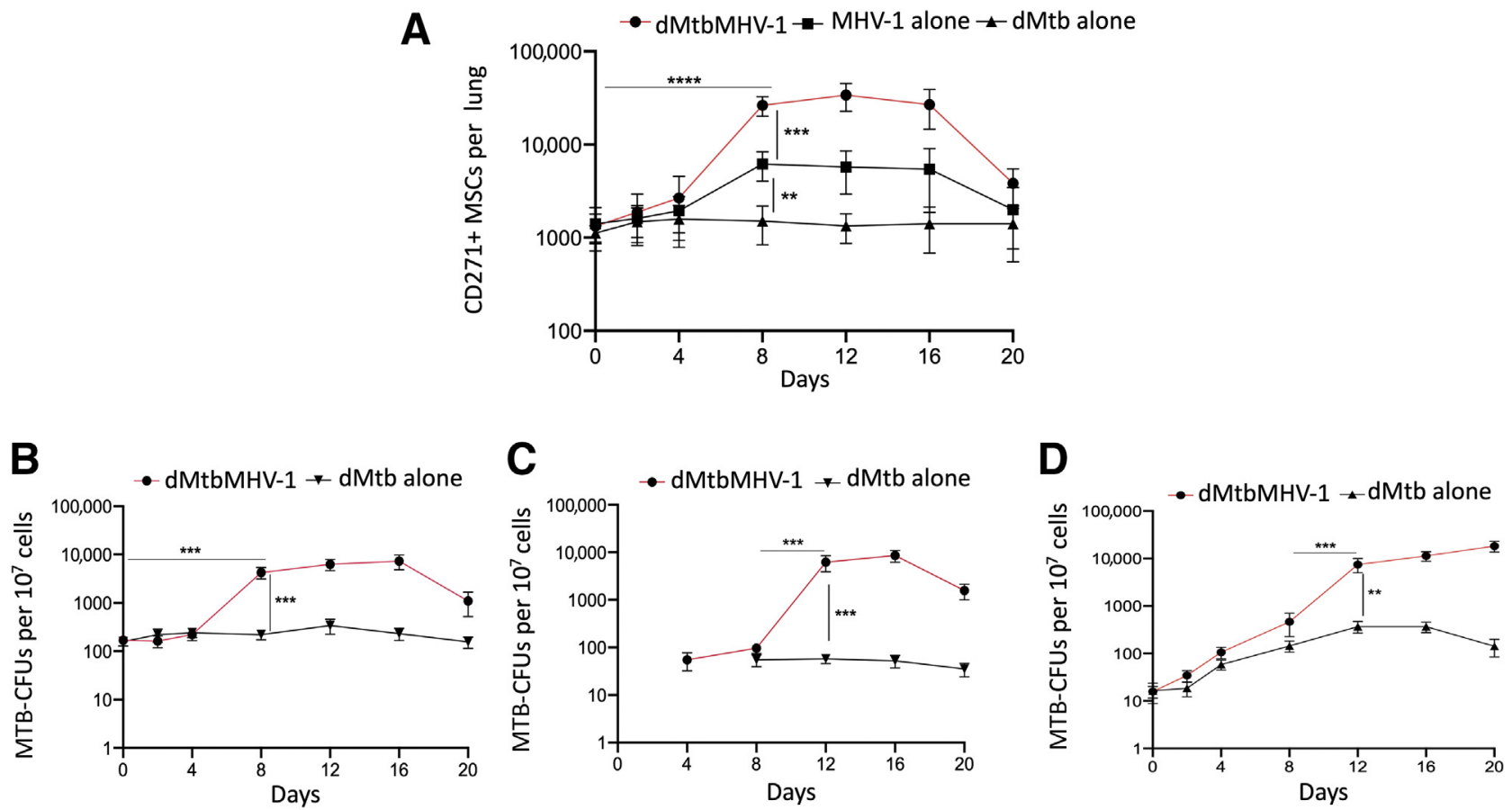

Figure 2 Mycobacterium tuberculosis (MTB) boosts murine hepatitis virus-1 (MHV-1)-mediated expansion of lung CD271 ${ }^{+}$mesenchymal stem cells (MSCs). A: Transient expansion of $\mathrm{CD}_{271}{ }^{+} \mathrm{CD} 45^{-}$MSCs in the lungs of MHV-1-infected and noninfected dormant MTB (dMTB) mice. The $y$ axis represents total number of immunomagnetically sorted $\mathrm{CD} 271^{+} / \mathrm{CD} 45^{-}$MSCs per lung; cells were subjected to immunomagnetic sorting to obtain CD45 and CD271 ${ }^{+}$MSCs, and the total numbers of cells were counted by trypan blue. B and C: Intracellular (B) and extracellular (C) MTB-colony-forming units (CFUs) obtained from CD271 ${ }^{+}$ MSCs of dMTB mice cultured in vitro for 8 hours. D: Intracellular MTB-CFU $/ 10^{7}$ of non-CD271 ${ }^{+}$lung cells (the leftover lung mononuclear cells after obtaining $\mathrm{CD} 271^{+} / \mathrm{CD} 45^{-}$MSCs by immunomagnetic sorting). Data represent means $\pm \mathrm{SEM}(\mathbf{A}-\mathbf{D}) . n=4$ independent experiments $(\mathbf{A}-\mathbf{D}) .{ }^{* *} P<0.01, * * * P<0.001$, and ${ }^{* * * * P}<0.0001$ (one-way analysis of variance with the Dunnett post hoc test).

$\mathrm{CD} 271^{+}$MSCs to the rest of the lung cells in the dMTBMHV-1 group probably occurred between days 8 and 12 , when the supernatant showed a sharp increase in MTBCFUs. Indeed, there was a corresponding 15 -fold increase of intracellular MTB-CFUs in the rest of the lung cells in vivo $(P<0.001)$, as shown in Figure $2 \mathrm{D}$. As expected, the dMTB alone group showed no significant evidence of $\mathrm{CD}_{271^{+}}$MSC expansion, intracellular MTB replication, and their extracellular release (Figure 2). Altogether, the results suggest that MHV-1 infection induces a transient expansion of MTB harboring CD271 ${ }^{+}$MSCs and pathogen's release into the extracellular space.

\section{MHV-1 Infection Activates the Altruistic Stem Cell-Mediated Innate Defense Mechanism}

The MHV-1 infection-induced transient expansion of lung CD271 $1^{+}$MSCs in both MTB harboring and control animal group led to studying the underlying molecular mechanism of expansion. MHV-1 infection may activate an ASCbased innate defense mechanism previously characterized in human embryonic stem cells, ${ }^{22}$ and in MSCs. ${ }^{18}$ Briefly, when a clone of stem cells, such as human embryonic stem cell colony, is threatened by hypoxia/oxidative stress-induced differentiation/apoptosis, a few of these cells reprogram to an enhanced stemness phenotype capable of not only surviving the stress, but also protecting the rest of the clone from the hypoxia/oxidative stress. This phenotype is characterized by distinct gene expression associated with HIF- $2 \alpha$ stemness pathway, ${ }^{22,37}$ and rapid expansion of the reprogrammed cells. These cells continue to maintain enhanced stemness phenotype for the next 2 weeks, and then activate $p 53$ to undergo differentiation/ apoptosis. During the expansion period, these cells exhibit altruistic behavior by secreting cytoprotective agents, such as glutathione, that enhance the fitness of the rest of the human embryonic stem cells. Thus, stem cells undergoing enhanced stemness reprogramming ${ }^{22}$ were characterized and termed altruistic stem cells. ${ }^{21,22}$ Interestingly, circulating MSCs having ASC-like characteristics ${ }^{24}$ were identified using defined criteria $^{34}$ (Figure 3A) in human subjects with cancer, ${ }^{24}$ suggesting the clinical significance of ASCs. Therefore, this ASC reprogramming may function as a putative innate defense system against invading pathogens that threaten the integrity of the stem cells residing in their niches. ${ }^{34}$ Thus, the findings of MHV1-mediated transient expansion of CD271 ${ }^{+}$MSCs may be part of the ASC defense mechanism against the virus. To investigate this possibility, the real-time quantitative PCR expression of genes associated with enhanced stemness phenotype (HIF-1 $\alpha, H I F-2 \alpha$, Sox2, Oct4, Nanog, ABCG2, $M D M 2, \quad G C L, \quad p 53$, and $p 21)$ was studied in 


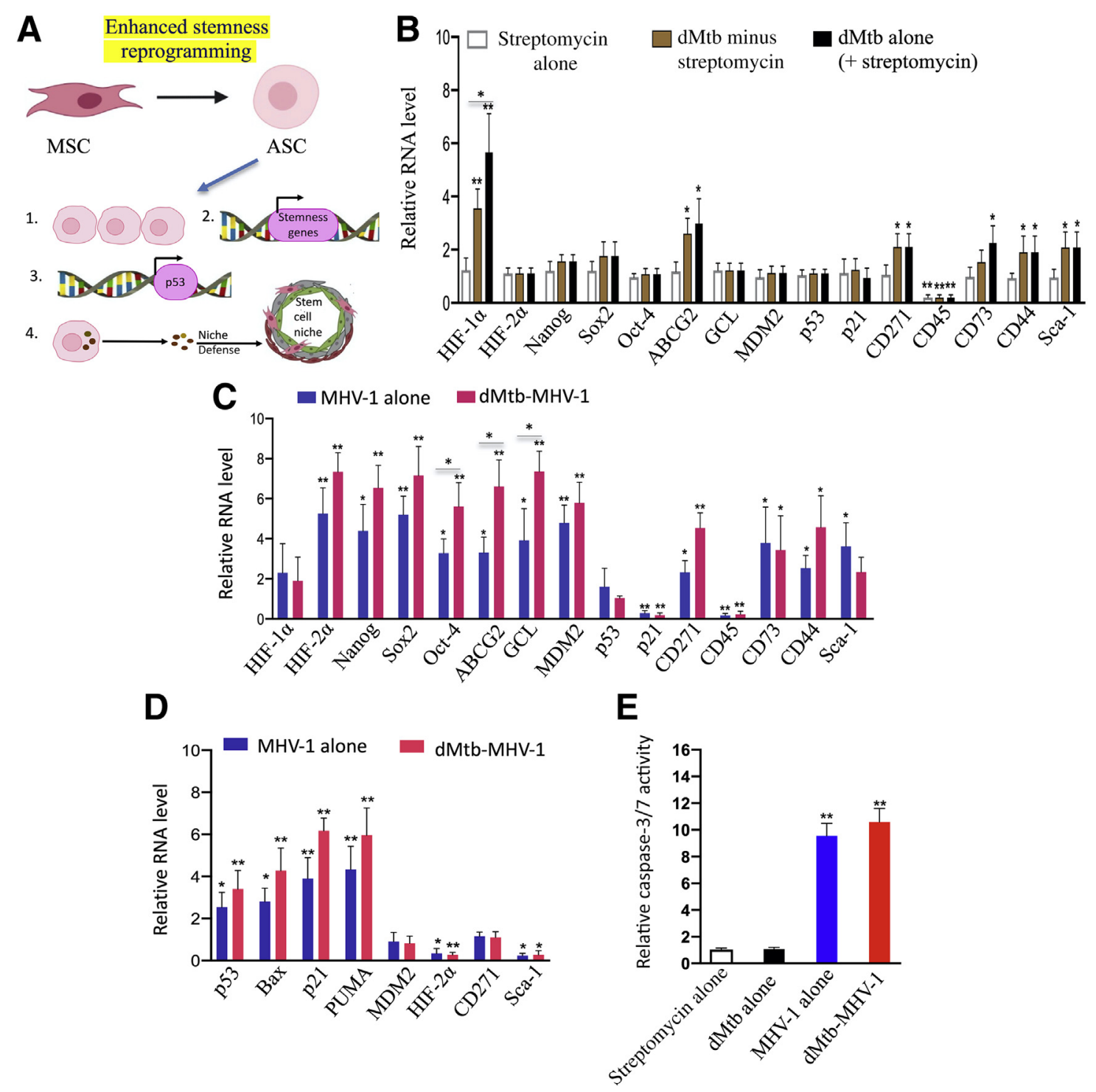

Figure 3 Murine hepatitis virus-1 (MHV-1) infection reprograms CD271 ${ }^{+}$mesenchymal stem cells (MSCs) to altruistic stem cell (ASC) phenotype. A: Phenotypic characteristics of ASCs or stem cells undergoing enhanced stemness reprogramming. 1. Transient expansion of ASCs. 2. Expression of genes associated with the enhanced stemness state. 3. After 2 weeks, activation of p53-induced genes of differentiation/apoptosis. 4. Stem cell niche-defense/ cytoprotection. B: Histogram showing the expression of MSC/ASC genes in the CD271+ MSCs of day 8 after streptomycin treatment. The real-time quantitative PCR ( $(\mathrm{PCR})$ data were compared with healthy mice to obtain fold change. C: Histogram showing the induction of enhanced stemness genes in the lung CD271 ${ }^{+}$MSCs of day 8 after MHV-1 infection. D: Histogram showing the induction of $p 53$-related genes of apoptosis/differentiation in lung CD271 ${ }^{+}$MSCs of day 20 after MHV-1 infection. The qPCR data are expressed as fold change compared with MTB-infection alone group. E: Increased caspase-3/7 activity in lung CD271 ${ }^{+}$MSCs of day 20 versus day 8 after MHV-1 infection. Data represent means \pm SEM $(\mathbf{B}-\mathbf{E}) . n=4$ independent experiments $(\mathbf{B}-\mathbf{E}) .{ }^{*} P<0.05$, ${ }^{* *} P<0.01$ (one-way analysis of variance with the Dunnett post hoc test). Control + streptomycin, healthy mice treated with streptomycin; dormant MTB (dMTB) alone (+ streptomycin), dMTB harboring mice treated with streptomycin; dMTB - streptomycin, dMTB minus Streptomycin, dMTB harboring mice not treated with streptomycin. ABCG2, ATP-binding cassette super-family G member 2; Bax, B cell lymphoma 2 associated X protein; CD271, cluster of differentiation 271; CD44, cluster of differentiation 44; CD45, cluster of differentiation 45; CD73: cluster of differentiation 73; GCL, glutamate cysteine ligase; Hif-1 alpha, hypoxia-inducible factor 1-alpha; Hif-2 alpha, hypoxia-inducible factor 2-alpha; MDM2, murine double minute 2 homolog; 0ct4, octamer-binding transcription factor 4; p53: tumor protein p53;p21, cyclin-dependent kinase inhibitor 1; PUMA, p53 upregulated modulator of apoptosis; Sca-1, stem cells antigen-1; Sox 2: sex-determining region Y-related HMG box 2.

the immunomagnetically sorted day $8 \mathrm{CD} 271^{+}$MSCs of MHV-1 alone and dMTBMHV-1 mice that showed expansion of the MSCs (Figure 2A). The dMTB alone (dMTB mice treated with streptomycin, but not infected with MHV-1), dMTB minus streptomycin (not treated with streptomycin and MHV-1), and streptomycin alone (control mice with streptomycin treatment) served as controls. The expression of genes expressed by MSCs (CD73, CD44, and Sca-1) and hematopoietic cells (CD45) was also evaluated (Figure 3, B and C). In the CD271 MSCs of dMTB mice, HIF-1 $\alpha$, ABCG2, Sca-1, and MSC related genes, CD44, CD271, and CD73 remained highly expressed (Figure 3B), as consistent with previous findings. ${ }^{17,18}$ Addition of streptomycin treatment showed 
1.6-fold higher expression of HIF-1 $\alpha \quad(P<0.05)$ (Figure 3B) in the CD271 ${ }^{+}$MSCs of dMTB mice (dMTB alone), but had no effect in the control mice (streptomycin alone). More importantly, MHV-1 infection in streptomycin alone (MHV-1 alone) and dMTB alone group (dMTBMHV-1) led to threefold to sevenfold marked induction of genes associated with enhanced stemness phenotype in the CD271 ${ }^{+}$MSCs (Figure 3C). Interestingly, in the dMTBMHV-1 group, several enhanced stemness phenotype or ASC reprogramming-related gene (Oct4, GCL, and $A B C G 2)$ expression was increased by twofold to threefold $(P<0.05)$ (Figure 3C) compared with MHV-1 alone group. The stemness of these CD271 ${ }^{+}$ MSCs was further characterized and, indeed, these cells expressed the MSC markers (CD73, CD44, and Sca-1), and did not express CD45 (Figure 3C). Thus, these findings suggest that MHV-1 infection led to ASC reprogramming of $\mathrm{CD} 271^{+} \mathrm{MSCs}$, and this reprogramming was also seen in the dMTB harboring mice, when infected with MHV-1. Further confirmation of the ASC reprogramming was obtained by demonstrating increased up-regulation of $p 53$ upstream genes involved in apoptosis, such as bax, $p 21$, and PUMA, and down-regulation of survival genes, such as $H I F-2 \alpha$, on day 20 (Figure 3D). The expression of Sca- 1 was down-regulated in these cells on day 20 (Figure 3D), suggesting differentiation. Thus, it appears that the transiently expanded CD $271^{+}$MSCs underwent differentiation and apoptosis on day 20 of MHV-1 infection, which was confirmed by performing a caspase-3/7 activity assay for apoptosis $^{22,37}$ (Figure 3E). Streptomycin treatment either in control (streptomycin alone) or in dMTB mice (dMTB alone) did not induce apoptosis of $\mathrm{CD} 271^{+}$MSCs
A

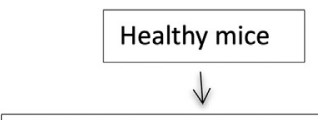

Type II alveolar epithelial cells (ATII cells)

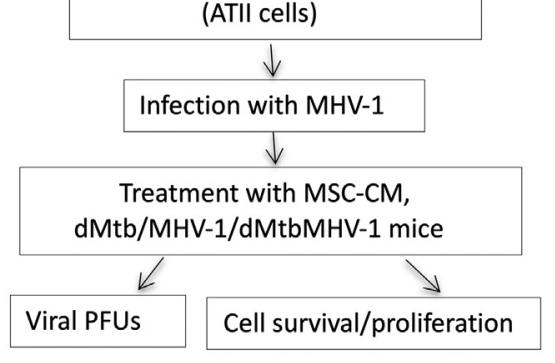

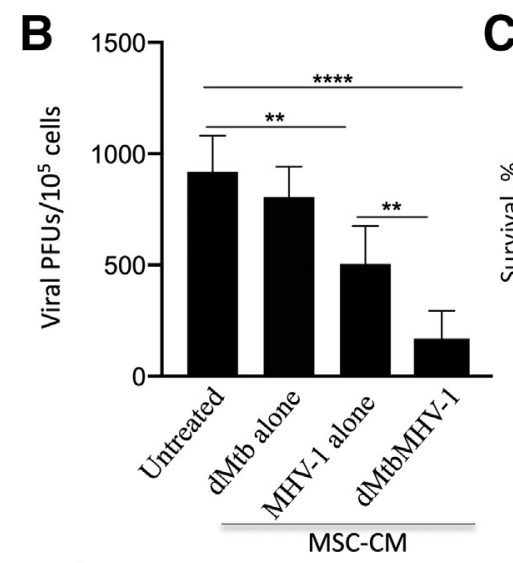
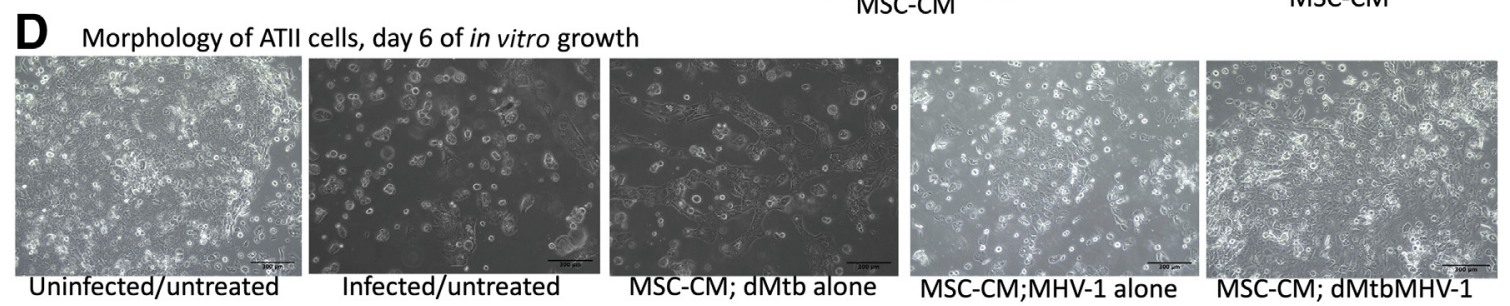

\section{E}

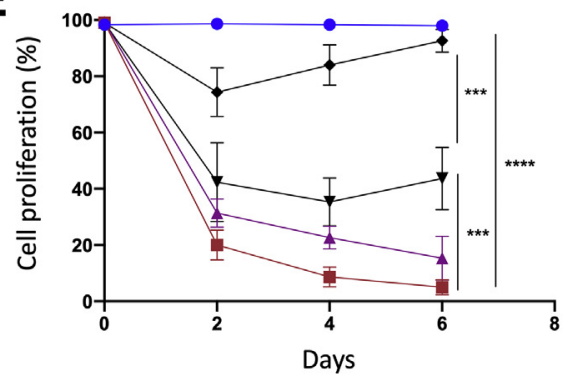

- Uninfected/untreated

- Infected/untreated

- MSC-CM; dMtb alone

$\rightarrow \mathrm{MSC}-\mathrm{CM} ; \mathrm{MHV}-1$ alone

- MSC-CM; dMtbMHV-1

Figure 4 Evidence for soluble factors of inhibitors of murine hepatitis virus-1 (MHV-1) infectivity and activators of cytoprotective effects in culture supernatants of reprogrammed CD271 ${ }^{+}$mesenchymal stem cells (MSCs). A: Schematic representation of experimental design for altruistic stem cell defense against MHV-1. B: The viral load of type II alveolar epithelial (ATII) cells was obtained after treatment with conditioned media (CM) from lung CD271 ${ }^{+}$MSCs. The CM of lung CD271 ${ }^{+}$MSCs (day 8 after infection) was obtained from all of the infected groups. C: Cell viability of the treated cells was obtained by trypan blue. The ATII cells not treated with CM served as the control. The CM from CD271+ MSCs of dormant Mycobacterium tuberculosis (dMTB)MHV-1 showed the lowest MHV-1-plaque-forming unit (PFU) in the ATII cells and highest live cell count. D: ATII cells under phase-contrast microscope after 6 days of treatment with CM of CD271 ${ }^{+}$MSCs obtained from mice lung. E: Alamar blue results of ATII cell proliferation. Data represent means \pm SEM $(\mathbf{B}, \mathbf{C}$, and E). $n=4$ independent experiments (B and $\mathbf{C}) ; n=3$ independent experiments (E). ${ }^{* *} P<0.01,{ }^{* * *} P<0.001$, and ${ }^{* * * * P}<0.0001$ ( $t$-test). Scale bar $=300 \mu \mathrm{m}(\mathbf{D})$. Original magnification, $\times 10$ (D). 
(Figure 3E). Activation of p53 upstream genes and associated apoptosis on day 20 also indicated the activation of the proposed ASC-based innate defense mechanism in the infected CD271 ${ }^{+}$MSCs against MHV-1.

The ASC-based defense against MHV-1-mediated viral load and cellular toxicity was confirmed in an in vitro viral infection assay (Figure 4A). The MHV-1 alone group derived $\mathrm{CM}$ of $\mathrm{CD} 271^{+}$MSCs on day 8 was exhibited significant twofold to fourfold cytoprotection of ATII cells against MHV-1 infection (Figure 4, B-E), providing evidence of ASC-mediated defense against MHV-1 (Figure 5). Notably, dMTB presence significantly boosted this ASCbased defense against MHV-1 in the in vitro viral infection assay, as the viral load was reduced by threefold (Figure 4B), whereas survival and proliferation of the ATII cells was increased by twofold and fourfold in the dMTB MHV-1 versus MHV-1 alone group, respectively (Figure 4, $\mathrm{C}$ and $\mathrm{E})$. These results further strengthen the proposed mechanism of ASC-mediated niche defense against MHV-1 and boosting of this defense in the dMTB harboring mice.

\section{Discussion}

The COVID-19 caused by SARS-CoV-2 has generated a global pandemic associated with substantial mortality and morbidity. Notably, a significant number of infected individuals have recovered. However, evidence for a possible host defense or antiviral mechanism against the virus has not yet been identified. Currently, several vaccines have been approved to combat the pandemic, ${ }^{43,44}$ although the efficacy of these vaccines may be compromised because of rapid mutation of the virus. In this context, it is important to understand the host defense mechanism against this disease for the development of better vaccine and/or treatment. Using a mouse coronavirus model, we demonstrate that lung MSCs activate a potential ASC-based defense mechanism against the virus, and this defense mechanism is further enhanced by dMTB residing inside the lung MSCs. Considering that MHV-1 infection mimics SARS-CoV-2 infection, and the dMTB mouse model mimics dMTB disease in humans, our study of MHV-1 and dMTB coinfection, as well as identification of ASC-based defense against MHV-1, may have significant clinical relevance to the ongoing COVID-19 pandemic.

To study the interaction of dormant MTB niche with coronavirus infection in the lung, a streptomycin-dependent auxotrophic strain of mutant $18 \mathrm{~b}$ mediated mouse model was used, where dMTB could be isolated from the CD271 ${ }^{+}$ MSC compartment of lung following 6 months of streptomycin starvation. ${ }^{17,42}$ In this mouse model, re-addition of streptomycin for 4 weeks after 6 months of withdrawal did not lead to dMTB reactivation as the lung MTB-CFUs in the non-MSC compartment did not significantly increase.

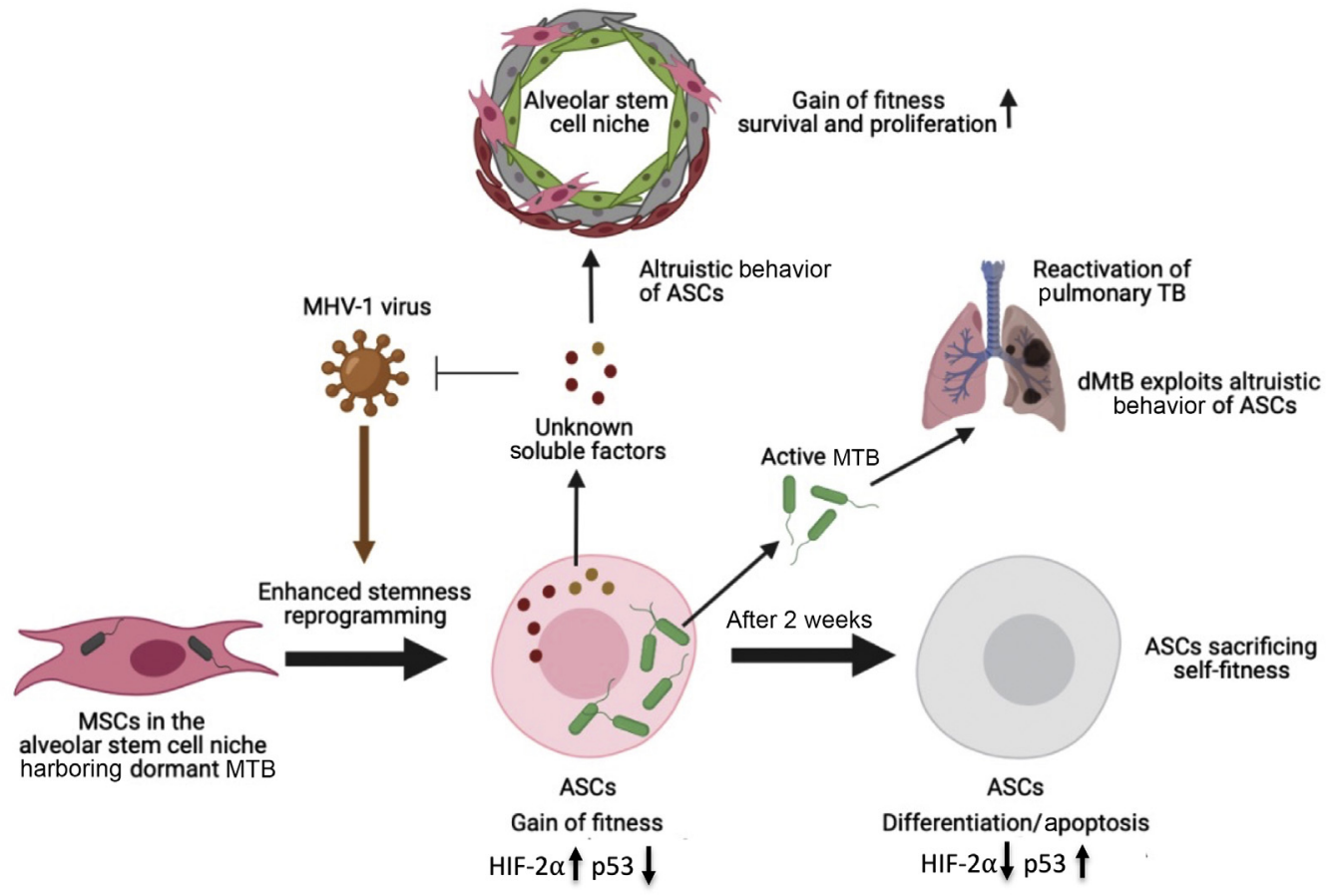

Figure 5 Schematic representation of the putative altruistic defense of stem cell niche against murine hepatitis virus-1 (MHV-1) infection in dormant Mycobacterium tuberculosis (dMTB) harboring mice. The viral infection induces enhanced stemness or altruistic stem cell (ASC) reprogramming of lung mesenchymal stem cells (MSCs), which then expands and secretes yet unknown secretory products/vesicles/antimicrobial peptides to enhance the group fitness of the type II alveolar cells present in the alveolar stem cell niche. The proliferating ASCs then undergo spontaneous apoptosis/differentiation by activating $p 53$ upstream genes involved in apoptosis, thus sacrificing self-fitness, similar to embryonic stem cell derived ASCs. The dMTB exploits this ASCbased stem cell niche defense for the reactivation of pulmonary tuberculosis (TB). HIF, hypoxia-inducible factor. 
Although a 20-fold increase in MTB-CFUs in non-MSC compartment was observed during first 2 weeks of streptomycin treatment, it was transient as MTB-CFUs gradually decreased in the next 2 weeks. Thus, the cumulative increase of MTB-CFUs was only threefold $(P>0.05)$ (Figure 1D), which is not enough to cause TB reactivation in lung. ${ }^{35}$ However, infection with MHV-1 in the presence of streptomycin caused sustained increase of MTB-CFUs in the non-MSC compartment of the lung by 3500-fold (Figure 1D), suggesting lung TB reactivation, as defined previously. ${ }^{35}$ More importantly, increase of MTB-CFUs in the non-MSC compartment was preceded by the transient expansion and enhanced stemness reprogramming of lung $\mathrm{CD} 271^{+}$MSCs, increase in intracellular replication of MTB CFUs, and their release into the extracellular space. These results suggest activated dMTB release from reprogrammed MSCs may have infected non-MSC lung cells to cause TB disease reactivation. Interestingly, although the potential contribution of non-MSC lung compartment in dMTB reactivation is most unlikely in this mouse model, as confirmed previously by in vivo transplantation assay, ${ }^{17}$ a sevenfold increase $(P<0.07)$ (Figure 2D) of MTB-CFUs was noted in the non-MSC lung compartment in the first 4 days of MHV-1 infection. Cross contamination-related presence of MSCs during immunomagnetic sorting may have contributed to the initial increase in MTB-CFUs. Future studies are required using flow cytometry sorting, as well as green fluorescent protein-labeled dMTB, to rule out any potential contribution of non-MSCs in the MHV-1-mediated reactivation of dMTB. Nevertheless, dMTB reactivation was associated with rapid decline of MHV-1 viral loads in the lung tissue, and associated direct antiviral activity of the culture supernatant of reprogrammed MSCs exhibiting enhanced stemness or ASC phenotype. More importantly, presence of dMTB in MSCs facilitated MSC to ASC reprogramming as indicated by a threefold to fourfold increase in CD271 ${ }^{+}$ MSC expansion and gene expressions of Oct4, GCL, and $A B C G 2$. Subsequently, after 2 weeks, these reprogrammed cells activated genes involved in differentiation/apoptosis, suggesting loss of fitness, indicating ASC phenotype of these cells. These results also indicate significant interaction between the two concurrent infections in the lung MSC compartment, and may provide valuable insight about MSC-mediated defense against MHV-1 infection, and how dMTB exploits the putative altruistic defense mechanisms for their reactivation.

Altruistic behavior has been reported in bacteria as a defense strategy against antibiotic exposure; a few members of bacteria sacrifice self-fitness to enhance group fitness, similar to kin selection noted in biological altruism. ${ }^{26,27,45}$ Whether the stem cells exert similar altruistic behavior as a defense strategy to protect their niche is not yet clear. We propose that stem cells exert altruistic behavior by the mechanism of stem cell altruism, as described previously, ${ }^{22,25,34}$ an idea inspired by Indian Vedic altruism theory of Jiva Upakarvada. ${ }^{23,55}$ The stem cell altruism could be defined as a fitness-defense mechanism that protects weak neighbors in their niche. ${ }^{34}$ This is in contrast to stem cell competition, which is a fitness-sensing mechanism that eliminates weak neighbors in the niche. ${ }^{46}$ Enhanced stemness reprogramming is a form of stem cell altruism that increases the fitness of surrounding tissues during the process of tissue regeneration. ${ }^{22,47}$ Furthermore, altruistic stem cells have been proposed in the context of stem cell niche-based innate defense mechanisms. ${ }^{17,18,34}$ The results of the present work provide first evidence of the potential role of ASC-based altruistic defense against viral infection of alveolar stem cell niches (Figure 5) composed of type II alveolar cells, MSCs, and endothelia progenitors. ${ }^{28,29}$ Cytoprotective activity of these ASCs was demonstrated in MHV-1-mediated toxicity of lung alveolar epithelial cells. The conditioned media of the ASCs (reprogrammed CD271 ${ }^{+}$MSCs) recovered from the MHV-1-infected mouse lung exhibited a direct antiviral and cytoprotective property on MHV-1-infected ATII cells by decreasing the viral load and increasing the ATII cell survival/ proliferation. After 2 weeks, these ASCs activated genes involved in apoptosis/differentiation, and exhibited increased caspase-3/7 activity, suggesting loss of fitness, thereby identiying putative MSC-derived ASCs. Hence, ASCmediated antiviral defense system for lung alveolar stem cell niche may be harnessed to develop novel therapeutic strategies against COVID-19. However, the current study has certain limitations. Future research is required to validate the altruistic nature of these reprogrammed CD271 ${ }^{+}$MSCs or ASCs, including the demonstration of potential activation of HIF-2 $\alpha$ stemness pathway, secretion of cytoprotective biomolecules that may act as a biofilm to shield the epithelial stem cell niche from the toxic effects of the virus, and inflammation. Furthermore, additional studies are required to assess the potential role of $\mathrm{CD} 271^{+}$ASCs to defend the lung alveolar stem cell niche, ${ }^{28,29,48}$ including potential secretion of antimicrobial peptides, such as cathelicidin hCAP-18/LL$3^{49}$ or miRNA. ${ }^{50-52}$ Taken together, this model of pathogeninduced enhanced stemness reprogramming of CD271 ${ }^{+}$ MSCs in the lung may serve as model to study the stem cell altruism, and its role in pathogen/host interaction. ${ }^{25}$

The adaptive immune response can play an important role in infection control of MHV-1 in C57BL/6 mice. ${ }^{32}$ However, this does not solely account for the resistance displayed by this mouse strain against the viral infection-associated morbidity and mortality. ${ }^{31}$ So far in this MHV-1 infection model and immune response, the role of ASC-based innate immunity has not been evaluated. These findings indicate that MHV-1 may induce an ASCmediated defense, as the conditioned media of the CD271 ASCs obtained from the MHV-1-infected mice exhibited direct antiviral activities. Further work is necessary to find a memory component in the ASC defense. The memory component of innate immunity, alias trained immunity, has recently been demonstrated in stem cells. ${ }^{48,53}$ For example, hematopoietic stem cells may undergo cell intrinsic 
changes, including metabolic as well as epigenetic modifications, to remember pathogenic insult ${ }^{48,53}$ and Bacille Calmette-Guerin-mediated trained immunity against viral infection. ${ }^{54}$ Although trained immunity of MSCs has not yet been demonstrated, ongoing work indicates that dMTB harboring MSCs may exhibit an HIF- $1 \alpha$ dependent trained immunity ${ }^{55}$; HIF- $1 \alpha$ is a mediator of trained immunity. ${ }^{48,53}$ We suggest that our findings of ASC-mediated innate immunity may play a key role in trained immunity. In this context, the findings of twofold increase of HIF- $1 \alpha$ and transient increase of MTB-CFUs in the dMTB mice following streptomycin treatment (Figure 3B) may indicate the triggering of trained immunity in the high HIF- $1 \alpha$ expressing CD271 ${ }^{+}$MSCs following streptomycin-induced dMTB reactivation. Future work is required to find if this putative trained immunity could be involved in the decline of MTB-CFUs in the dMTB alone mice following initial increase in the first 2 weeks of streptomycin treatment. Thus, our model of MTB and MHV-1 co-infection in mice may be a useful model to study potential MSC/ASC-based trained immunity against coronavirus.

Recent reports suggest that subjects with TB increase the susceptibility and severity of SARS-CoV-2 ${ }^{45,56}$ In contrast, the current findings suggest that MTB reactivation reduces the viral load in lung. However, this study is based on a mouse model, and ther results need to be confirmed using human clinical samples. Therefore, MTB reactivation in the lungs of COVID-19 subjects is currently being studied. The presence of circulating ASC is being investigated in these subjects as potential evidence for SARS-CoV-2-mediated ASC defense mechanism by adopting a method of the isolation of circulating ASCs, as described previously. ${ }^{24}$

In summary, we found that MHV-1 infection induces dMTB loaded MSCs to reprogram to ASCs, thus activating the ASC defense mechanism and diminishing the MHV-1 infection. Knowledge of immunity to SARS-CoV-2 is still at a preliminary stage. Thus, this study may help in understand how MSCs inducing ASC defense mechanism may help in combating the viral load in the host, thereby helping in developing a possible cure for COVID-19.

\section{Acknowledgments}

We thank the members of Department of Stem Cell and Infectious Diseases, KaviKrishna Laboratory, Guwahati Biotech Park, Indian Institute of Technology (Guwahati, India), Department of Stem Cell and Infection, Thoreau Lab for Global Health, University of Massachusetts (Lowell, MA), and Department of Bioengineering and Technology, Gauhati University (Guwahati, India).

\section{Author Contributions}

B.D. initiated and designed the study. B.D., L.P., S.G., B.P., S.B., S.S., and J.T. performed the in vitro and in vivo experiments. B.D., L.P., S.G., and B.P. analyzed data. H.Y. provided materials and reagents. D.B. provided access to animal facility and obtained ethical permissions. B.D., L.P., and S.G. wrote the manuscript B.D., L.P., and H.Y. edited the manuscript.

\section{References}

1. Walaza S, Tempia S, Dawood H, Variava E, Wolter N, Dreyer A, Moyes J, Von Mollendorf C, McMorrow M, Von Gottberg A, Haffejee S, Venter M, Treurnicht FK, Hellferscee O, Martinson NA, Ismail $\mathrm{N}$, Cohen $\mathrm{C}$ : The impact of influenza and tuberculosis interaction on mortality among individuals aged $>/=15$ years hospitalized with severe respiratory illness in South Africa, 20102016. Open Forum Infect Dis 2019, 6:ofz020

2. Oei W, Nishiura H: The relationship between tuberculosis and influenza death during the influenza (H1N1) pandemic from 1918-19. Comput Math Methods Med 2012, 2012:124861

3. Walaza S, Cohen C, Tempia S, Moyes J, Nguweneza A, Madhi SA, McMorrow M, Cohen AL: Influenza and tuberculosis co-infection: a systematic review. Influenza Other Respir Viruses 2020, 14:77-91

4. Low JG, Lee CC, Leo YS: Severe acute respiratory syndrome and pulmonary tuberculosis. Clin Infect Dis 2004, 38:e123-e125

5. Alfaraj SH, Al-Tawfiq JA, Altuwaijri TA, Memish ZA: Middle east respiratory syndrome coronavirus and pulmonary tuberculosis coinfection: implications for infection control. Intervirology 2017, 60: $53-55$

6. Redford PS, Mayer-Barber KD, McNab FW, Stavropoulos E, Wack A, Sher A, O'Garra A: Influenza A virus impairs control of Mycobacterium tuberculosis coinfection through a type I interferon receptor-dependent pathway. J Infect Dis 2014, 209:270-274

7. de Paus RA, van Crevel $R$, van Beek R, Sahiratmadja E, Alisjahbana B, Marzuki S, Rimmelzwaan GF, van Dissel JT, Ottenhoff TH, van de Vosse E: The influence of influenza virus infections on the development of tuberculosis. Tuberculosis (Edinb) 2013, 93:338-342

8. Mamelund SE, Dimka J: Tuberculosis as a risk factor for 1918 influenza pandemic outcomes. Trop Med Infect Dis 2019, 4:2

9. Walaza S, Tempia S, Dawood H, Variava E, Moyes J, Cohen AL, Wolter N, Groome M, von Mollendorf C, Kahn K, Pretorius M, Venter M, Madhi SA, Cohen C: Influenza virus infection is associated with increased risk of death amongst patients hospitalized with confirmed pulmonary tuberculosis in South Africa, 2010-2011. BMC Infect Dis 2015, 15:26

10. Pandemic Influenza A (H1N1) 2009: Considerations for Tuberculosis Care Services, Paul Nunn (Coordinator) and Dennis Falzon (Medical Officer). Geneva, Switzerland, Stop TB Department, World Health Organisation, 2009

11. Park Y, Chin BS, Han SH, Yun Y, Kim YJ, Choi JY, Kim CO, Song YG, Kim JM: Pandemic influenza (H1N1) and Mycobacterium tuberculosis co-infection. Tuberc Respir Dis (Seoul) 2014, 76:84-87

12. Volkert M, Pierce C, Horsfall FL, Dubos RJ: The enhancing effect of concurrent infection with pneumotropic viruses on pulmonary tuberculosis in mice. J Exp Med 1947, 86:203-214

13. Co DO, Hogan LH, Karman J, Heninger E, Vang S, Wells K, Kawaoka Y, Sandor M: Interactions between T cells responding to concurrent mycobacterial and influenza infections. J Immunol 2006, $177: 8456-8465$

14. Okabayashi T, Kariwa H, Yokota S, Iki S, Indoh T, Yokosawa N Takashima I, Tsutsumi H, Fujii N: Cytokine regulation in SARS coronavirus infection compared to other respiratory virus infections. J Med Virol 2006, 78:417-424

15. Kaufmann SH, Dorhoi A: Inflammation in tuberculosis: interactions, imbalances and interventions. Curr Opin Immunol 2013, 25:441-449 
16. Beamer G, Major S, Das B, Campos-Neto A: Bone marrow mesenchymal stem cells provide an antibiotic-protective niche for persistent viable Mycobacterium tuberculosis that survive antibiotic treatment. Am J Pathol 2014, 184:3170-3175

17. Das B, Kashino SS, Pulu I, Kalita D, Swami V, Yeger H, Felsher DW, Campos-Neto A: CD271(+) bone marrow mesenchymal stem cells may provide a niche for dormant Mycobacterium tuberculosis. Sci Transl Med 2013, 5:170-170ra13

18. Garhyan J, Bhuyan S, Pulu I, Kalita D, Das B, Bhatnagar R: Preclinical and clinical evidence of Mycobacterium tuberculosis persistence in the hypoxic niche of bone marrow mesenchymal stem cells after therapy. Am J Pathol 2015, 185:1924-1934

19. Jones E, McGonagle D: Human bone marrow mesenchymal stem cells in vivo. Rheumatology (Oxford) 2008, 47:126-131

20. Spaeth EL, Kidd S, Marini FC: Tracking inflammation-induced mobilization of mesenchymal stem cells. Methods Mol Biol 2012, 904:173-190

21. BD: Altruistic stem cells and cancer stem cells. Edited by Rajasekhar VK. In Cancer Stem Cells. Hoboken, NJ: John Wiley \& Sons, 2014. pp. 89-106

22. Das B, Bayat-Mokhtari R, Tsui M, Lotfi S, Tsuchida R, Felsher DW, Yeger H: HIF-2alpha suppresses p53 to enhance the stemness and regenerative potential of human embryonic stem cells. Stem Cells 2012, 30:1685-1695

23. Das B: The Science Behind Squalene: The Human Antioxidant. Toronto, Canada, Toronto Medical Pub., for the International Council for Bionutrient Research, 2000. pp. 187

24. Talukdar JBR, Garhyan J, Pal B, Sandhya S, Gayan S, Sarma A, Mokhtari RB, Li H, Phukan J, Tasabehji W, Bhuyan S, Kataki AC, Tsuchida R, Yeger H, Baishya D, Das B: Migratory cancer side population cells induces stem cell altruism in bone marrow mesenchymal stem cells to resist therapy, and enhance tumorigenic potential of non-tumorigenic cells. In: Proceedings of the 107th Annual Meeting of the American Association for Cancer Research, New Orleans, LA Philadelphia (PA) 2016, AACR. Cancer Res 2016, 76(Suppl), Abstract 920

25. Pathak L, Das B: Initiation of post-primary tuberculosis of the lungs: exploring the secret role of bone marrow derived stem cells. Front Immunol 2021, 11:594572

26. Youk H, van Oudenaarden A: Microbiology: altruistic defence. Nature 2010, 467:34-35

27. West SA, Griffin AS, Gardner A, Diggle SP: Social evolution theory for microorganisms. Nat Rev Microbiol 2006, 4:597-607

28. Lee JW, Fang X, Krasnodembskaya A, Howard JP, Matthay MA: Concise review: mesenchymal stem cells for acute lung injury: role of paracrine soluble factors. Stem Cells 2011, 29:913-919

29. Zhu H, Xiong Y, Xia Y, Zhang R, Tian D, Wang T, Dai J, Wang L, Yao H, Jiang H, Yang K, Liu E, Shi Y, Fu Z, Gao L, Zou L: Therapeutic effects of human umbilical cord-derived mesenchymal stem cells in acute lung injury mice. Sci Rep $2017,7: 39889$

30. De Albuquerque N, Baig E, Ma X, Zhang J, He W, Rowe A, Habal M, Liu M, Shalev I, Downey GP, Gorczynski R, Butany J, Leibowitz J, Weiss SR, McGilvray ID, Phillips MJ, Fish EN, Levy GA: Murine hepatitis virus strain 1 produces a clinically relevant model of severe acute respiratory syndrome in A/J mice. J Virol 2006, 80:10382-10394

31. Khanolkar A, Fulton RB, Epping LL, Pham NL, Tifrea D, Varga SM, Harty JT: T cell epitope specificity and pathogenesis of mouse hepatitis virus-1-induced disease in susceptible and resistant hosts. J Immunol 2010, 185:1132-1141

32. Khanolkar A, Hartwig SM, Haag BA, Meyerholz DK, Harty JT, Varga SM: Toll-like receptor 4 deficiency increases disease and mortality after mouse hepatitis virus type 1 infection of susceptible C3H mice. J Virol 2009, 83:8946-8956

33. Khanolkar A, Hartwig SM, Haag BA, Meyerholz DK, Epping LL, Haring JS, Varga SM, Harty JT: Protective and pathologic roles of the immune response to mouse hepatitis virus type 1: implications for severe acute respiratory syndrome. J Virol 2009, 83: $9258-9272$

34. Pal B, Das B: In vitro culture of naive human bone marrow mesenchymal stem cells: a stemness based approach. Front Cell Dev Biol $2017,5: 69$

35. Scanga CA, Mohan VP, Joseph H, Yu K, Chan J, Flynn JL: Reactivation of latent tuberculosis: variations on the Cornell murine model. Infect Immun 1999, 67:4531-4538

36. Leibowitz J, Kaufman G, Liu P: Coronaviruses: propagation, quantification, storage, and construction of recombinant mouse hepatitis virus. Curr Protoc Microbiol 2011. Chapter 15:Unit 15E.1

37. Das B, Pal B, Bhuyan R, Li H, Sarma A, Gayan S, Talukdar J, Sandhya S, Bhuyan S, Gogoi G, Gouw AM, Baishya D, Gotlib JR, Kataki AC, Felsher DW: MYC regulates the HIF2alpha stemness pathway via Nanog and Sox 2 to maintain self-renewal in cancer stem cells versus non-stem cancer cells. Cancer Res 2019, 79: 4015-4025

38. Kebaabetswe LP, Haick AK, Miura TA: Differentiated phenotypes of primary murine alveolar epithelial cells and their susceptibility to infection by respiratory viruses. Virus Res 2013 , 175:110-119

39. Pal BBS, Baishya D, Das B: Oral cancer stem cells modulate Fusobacterium nucleatum to acquire the capability to induce tumor stemness switch. In: Proceedings of the American Association for Cancer Research Annual Meeting, Chicago, IL Philadelphia (PA) 2018, AACR. Cancer Res 2018, 78(Suppl), Abstract 3064

40. Bayat Mokhtari R, Baluch N, Ka Hon Tsui M, Kumar S, Homayouni TS, Aitken K, Das B, Baruchel S, Yeger H: Acetazolamide potentiates the anti-tumor potential of HDACi, MS-275, in neuroblastoma. BMC Cancer 2017, 17:156

41. He Y, Das B, Baruchel S, Kumar P, Wiebe L, Reilly RM: Meta[123I]iodobenzylguanidine is selectively radiotoxic to neuroblastoma cells at concentrations that spare cells of haematopoietic lineage. Nucl Med Commun 2004, 25:1125-1130

42. Kashino SS, Ovendale P, Izzo A, Campos-Neto A: Unique model of dormant infection for tuberculosis vaccine development. Clin Vaccine Immunol 2006, 13:1014-1021

43. Eastman RT, Roth JS, Brimacombe KR, Simeonov A, Shen M, Patnaik S, Hall MD: Remdesivir: a review of its discovery and development leading to emergency use authorization for treatment of COVID-19 [Erratum appeared in ACS Cent Sci. 2020;6:1009]. ACS Cent Sci 2020, 6:672-683

44. Kaur SP, Gupta V: COVID-19 vaccine: a comprehensive status report. Virus Res 2020, 288:198114

45. Lee HH, Molla MN, Cantor CR, Collins JJ: Bacterial charity work leads to population-wide resistance. Nature 2010, 467: $82-85$

46. Di Gregorio A, Bowling S, Rodriguez TA: Cell competition and its role in the regulation of cell fitness from development to cancer. Dev Cell 2016, 38:621-634

47. Cummins EP: HIF-2 $\alpha$ - a mediator of stem cell altruism? Stem Cell Res Ther 2012, 3:52

48. Naik S, Larsen SB, Cowley CJ, Fuchs E: Two to tango: dialog between immunity and stem cells in health and disease. Cell 2018, 175:908-920

49. Krasnodembskaya A, Song Y, Fang X, Gupta N, Serikov V, Lee JW, Matthay MA: Antibacterial effect of human mesenchymal stem cells is mediated in part from secretion of the antimicrobial peptide LL-37. Stem Cells 2010, 28:2229-2238

50. Qian X, Xu C, Fang S, Zhao P, Wang Y, Liu H, Yuan W, Qi Z: Exosomal microRNAs derived from umbilical mesenchymal stem cells inhibit hepatitis C virus infection. Stem Cells Transl Med 2016, 5:1190-1203

51. Khatri M, Richardson LA, Meulia T: Mesenchymal stem cell-derived extracellular vesicles attenuate influenza virus-induced acute lung injury in a pig model. Stem Cell Res Ther 2018, 9:1-17 
52. Yang K, Wang J, Wu M, Li M, Wang Y, Huang X: Mesenchymal stem cells detect and defend against gammaherpesvirus infection via the cGAS-STING pathway. Sci Rep 2015, 5:7820

53. Netea MG, Domínguez-Andrés J, Barreiro LB, Chavakis T, Divangahi M, Fuchs E, Joosten LAB, van der Meer JWM, Mhlanga MM, Mulder WJM, Riksen NP, Schlitzer A, Schultze JL, Stabell Benn C, Sun JC, Xavier RJ, Latz E: Defining trained immunity and its role in health and disease. Nat Rev Immunol 2020, 20:375-388

54. Arts RJW, Moorlag S, Novakovic B, Li Y, Wang SY, Oosting M, Kumar V, Xavier RJ, Wijmenga C, Joosten LAB, Reusken C, Benn CS, Aaby P, Koopmans MP, Stunnenberg HG, van Crevel R, Netea MG: BCG vaccination protects against experimental viral infection in humans through the induction of cytokines associated with trained immunity. Cell Host Microbe 2018, 23:89-100.e5

55. Das B, Pathak L, Gayan S, Pal B, Saikia PJ, Baishya T, Das NR, Das R, Maral M, Mahanta R, Bhuyan S, Gautam P, Talukdar J, Sandhya S, Kalita D, Swami V, Das KR, Borah DK, Ghora J, Pulu I: Stem cell basis of a host driven transmission of antigen packed aerosols: a novel mechanism of natural vaccination for tuberculosis. bioRxiv 2020. doi:10.1101/2020.11.14.382572

56. Liu Y, Bi L, Chen Y, Wang Y, Fleming J, Yu Y, Gu Y, Liu C, Fan L, Wang X, Cheng M: Active or latent tuberculosis increases susceptibility to COVID-19 and disease severity. medRxiv 2020. doi: $10.1101 / 2020.03 .10 .20033795$ 